\title{
TRABAJANDO PSICOANALITICAMENTE EN Y CON INSTITUCIONES. ESTRUCTURAS Y DINAMISMOS
}

Eliseo Miguel González Regadas Licenciado en Psicología, UDELAR Miembro Habilitante de AUDEPP

Ex profesor titular del IUPA Correo electrónico: eliseogon@hotmail.com ORCID: 0000-002-6032-2875 


\section{Resumen}

El presente artículo propone una articulación al servicio de la tarea terapéutica de encuadres y dispositivos diferentes, por un lado, y, por otro, de dinamismos transferenciales y circulantes movilizados durante el proceso.

Examinamos lo circulante y lo transferencial atravesando diferentes dispositivos y encuadres y viendo sus dinamismos y la relación que hay entre unos y otros.

Palabras clave: circulante, dispositivo asistencial, transferencia.

\section{Psychoanalytic work with and in institutions.}

\section{Structures and dynamics}

\section{Abstract}

This article proposes an articulation at the service of different frames and devices, on the one hand, and, on the other, of transference and circulating dynamisms mobilized during the process.

We examine the circulating and the transferential by going through different devices and frames and examining their dynamics and the relationship between them.

Keywords: circulation, assisive device, transference. 


\section{ENCUADRES Y DISPOSITIVOS DIFERENTES ${ }^{1}$}

El tema de los encuadres y sus particularidades, para que pueda discurrir un proceso psicoanalítico, ha sido una de las preocupaciones constantes de nuestra disciplina desde sus mismos orígenes. Quien hizo una sistematización de este tópico en los años setenta fue José Bleger (1976), quien consideró al encuadre como una institución. Cornelius Castoriadis (1989) lo llamó lo instituido, para diferenciarlo de lo instituyente. Queremos hacer un aporte al tema desde la perspectiva de los dinamismos que acontecen en diferentes espacios y su posible articulación desde una óptica teórico-clínica. Los dinamismos, a su vez, dependen de los encuadres instituidos y de sus avatares en el curso de los tiempos insumidos en los procesos que allí se desarrollan. Cómo distinguir y articular fenómenos parecidos, pero diferentes, y sus implicancias para un trabajo psicoanalítico en y con instituciones es lo que desarrollaremos.

En un trabajo publicado en 1992 abordamos el tema de las transferencias referidas a lo psicótico (González Regadas, 1992). Consideramos que, para entender, participar o intervenir en una institución, es central poder identificar lo que se desplaza en un momento dado, ya sea como dato, rumor o imaginario; y que sirve de vehículo cargado de sentido y

1 A los efectos de este trabajo, vamos a definir encuadre como el marco regulatorio en el que va a realizarse el proceso psicoanalítico y que es trasmitido por el o los terapeutas al o a los pacientes. Por otra parte, los dispositivos son las modalidades de abordaje empleadas por los terapeutas —o equipos_ - para llevar adelante el proceso. A modo de ejemplo: dispositivos individuales, grupales, familiares, de pareja, en instituciones, etc. Cada dispositivo, a su vez, tiene su propio encuadre. 
productor de efectos para las personas implicadas. Estos efectos y sentido pueden generar angustia y provocar situaciones conflictivas. Se trata de un típico fenómeno colectivo al que llamamos circulante, al que no hay que ver como algo personal. Ciertamente, son los individuos los que ponen en marcha el circulante, pero este es transpersonal. Hay siempre un proceso colectivo alusivo a situaciones propias de la vida institucional. ${ }^{2}$ Las instituciones constituyen un marco relativamente estable para los diferentes dispositivos albergados por ella, donde se dan procesos diferentes que están íntimamente interconectados y que podemos analizar y considerar en su profunda interrelación. Sabemos que los espacios utilizados, los tiempos, las modalidades de pago, etc., condicionan — de una u otra forma - los procesos terapéuticos. La supuesta «invariante» del encuadre o los encuadres en la historia del psicoanálisis se ha convertido en una variable más a considerar en cada momento histórico, en cada sociedad concreta y en cada cultura particular. Las instituciones — sobre todo las del psicoanálisis- deben ser analizadas a la par de los procesos asistenciales allí desarrollados para dar cumplimiento a su tarea primaria.

\section{DINAMISMOS EN DISPOSITIVOS Y ENCUADRES: LO CIRCULANTE Y LO TRANSFERENCIAL}

Hemos visto que lo que circula a título de informaciones, tal como comunicar un atraso en el pago de los salarios, afecta la tarea primaria. Lo circulante, ya sea un rumor o una información verificable, discurre por los registros consciente y preconsciente, mientras que los afectos y efectos asociados que estos evocan y provocan son inconscientes para los afectados directamente (Castoriadis, 1989).

2 Esto se parece mucho a los fenómenos de contagio descritos en los siglos xix y xx. 
La cuestión sobre diferenciar lo circulante de lo transferencial es importante en lo referente a lo metapsicológico. Una vez distinguidos e identificados, ulteriormente es necesario ver las posibilidades de su articulación para así ampliar la comprensión de lo que está aconteciendo en un momento dado en espacios distintos. Reviste gran interés práctico porque pone en evidencia cómo ambos fenómenos pueden potenciarse recíprocamente. Lo circulante puede dinamizar lo transferencial y aspectos referidos a lo transferencial pueden llegar a poner en marcha un circulante. Lo circulante está siempre vinculado con una vulnerabilidad específica - ya sea del colectivo en cuestión, de un grupo que allí funciona o de un individuo en particular-, que produce angustia y sufrimiento entre los implicados, que son parte de una institución y de los grupos humanos que allí funcionan.

Un circulante puede promover el surgimiento de transferencias y las situaciones transferenciales también movilizan circulantes; esto porque nuestros psiquismos no son mónadas, sino que están interconectados y abiertos al mundo relacional y social. Por ello, podemos hablar de un circulante transferencial o bien de lo transferencial circulando. Si el dinamismo surge del registro inconsciente de un sujeto y logra un eco o resonancia en el contexto grupal, familiar o social, podemos identificarlo como un circulante transferencial. Si se trata de un rumor proveniente de un espacio social, grupal o institucional, pensaríamos en un circulante. Este rumor circulante puede evocar reacciones transferenciales en los sujetos y llevarlos a darle un sentido singular e idiosincrásico al hecho. Por ejemplo, puedo vivenciar lo que acontece como un poder suprapersonal que controla todo y que, en este momento, me está haciendo un daño en represalia por algo que hice.

La importancia de examinar ambos aspectos en simultáneo y ver posibilidades de articulación o de disyunción nos da una perspectiva más 
amplia del proceso psicoterapéutico que estamos explorando. ${ }^{3}$ Como en todo lo humano, siempre hay zonas grises que dan lugar a un eventual solapamiento de ambos fenómenos, que responden a una etiología diferente y que afectan a individuos y colectivos en momentos problemáticos. Ciertamente, nos hemos preguntado si llamarlos circulantes o si catalogarlos a todos como trasferencias. ${ }^{4}$ ¿Es de utilidad para entender la clínica institucional y sus matices? Nuestra respuesta es afirmativa; para ello, nos apoyamos en el concepto freudiano de transcripción, ${ }^{5}$ ya mencionado en el borrador de su Proyecto de psicología (Freud, 1991b) y sostenido hasta el final de su vida en el Esquema del psicoanálisis (Freud, 1980). Cuando algo pasa de un espacio a otro sufre un cambio, una transformación, se transcribe en el otro espacio acorde a las leyes que lo rigen. El juego entre lo transferencial y lo circulante puede tornarse algo muy sutil en ciertos momentos en que se da una concordancia y una sincronía entre fenómenos que discurren por tópicas distintas. Apelaremos a un ejemplo.

3 Para ver una situación que articula momentos, espacios y recursos técnicos diferentes, sugerimos la lectura de nuestra presentación al Congreso Internacional de Psicoterapia realizado en Lima en 2009 y en las Jornadas Más allá del consultorio III, de AUDEPP, en 2010 (González Regadas, 2009-2010).

4 El concepto de circulante tiene parentesco con el que los franceses llamaron transversalidad o atravesamiento para aludir al campo de las instituciones (Guattari, 1976). Al hablar de circulantes transferenciales o de transferencias circulantes por los espacios institucionales, nos referimos a estas zonas grises donde hay superposiciones de ambos fenómenos.

5 En la famosa Carta 52 ya Freud (1991a) nos decía que el pasaje de un registro mental a otro implica una transcripción que va a seguir las leyes del nuevo registro (Freud, 1979, 1980). A modo de ejemplo, la diferencia entre la representación de cosa en lo inconsciente y en el sistema preconsciente o consciente es que hay una transcripción que incluye una representación de palabra. Por analogía usamos transcripción para referimos al pasaje del campo de lo social al de lo institucional-grupal y de allí al de lo familiar. El fenómeno transferido desde el inconsciente individual sufre una transcripción cuando pasa de un espacio a otro, ya que cada uno de ellos posee lógicas diferentes que les son propias. 


\section{LO SUCIO, LA SUCIEDAD: CIRCULACIÓN Y TRANSFERENCIAS}

La Sra. H. estaba internada en un hospital psiquiátrico desde hacía muchos años. Un terapeuta joven, a quien yo supervisaba, se dispuso a tomarla en psicoterapia «para aprender». Iba todos los días al hospital psiquiátrico con el propósito de tener encuentros con fines psicoterapéuticos con esa persona.

En el primero, la Sra. H. se presentó «sucia», «con olor a mierda», semidesnuda y ubicada en un rincón de la sala. Tanto su cama como los objetos que estaban sobre ella «eran un revoltijo, un nido de caranchos». El terapeuta comentó que sus compañeros del equipo que trabajaba en sala haciendo rehabilitación comenzaron a llamarlo «el kamikaze».

Durante un encuentro conmigo comentó, en un momento, que experimentaba una sensación de inoperancia: «Me siento muerto cada vez que terminamos la sesión y salgo de allí, soy un despojo humano, un monstruo. No sé por qué, a pesar de todo, sigo atendiéndola». Después, como al pasar, agregó que, cuando se encontró con la madre de la paciente en una visita al hospital, descubrió en ella «una mirada asesina que recorrió mi médula de arriba abajo produciéndome escalofrío y parálisis. De pronto, experimenté un impulso incoercible de matarla. ¡Soy un monstruo, todo esto es una locura!». Hubo un silencio prolongado entre nosotros para recuperarnos de estos comentarios tan cargados.

Le propuse explorar estas vivencias. Evocó dos situaciones acontecidas durante las sesiones. En la primera, la paciente le decía desgarrada por la angustia: «Mi madre me dijo una vez: "Sos una bestia; estás totalmente desquiciada, tu lugar es el manicomio"». La madre de la Sra. $\mathrm{H}$. de joven había trabajado en un geriátrico y la paciente, una niña pequeña, recordaba oírle decir: «Trabajo con muertos, desechos humanos pudriéndose allí». La segunda asociación aportada fue lo que sintió, en 
otra ocasión, al entrar a la sala donde la Sra. H estaba internada: «Sentí tanto olor a podrido, algo descompuesto, que me dieron náuseas y tuve que salir a vomitar». A su regreso, la Sra. H lo miró y le dijo: «Váyase, por favor, usted es demasiado joven y bueno; lo de aquí le hace mucho mal». El terapeuta, angustiado y llorando, me comentó: «No sirvo para trabajar en un hospital psiquiátrico; los internados son, realmente, desechos humanos». Un día, el psicoterapeuta fue a la casa de su madre, luego de una sesión con la Sra. H, y ella le comentó muy preocupada: «Te estás volviendo un monstruo yendo a trabajar a ese lugar. Cuando venís de allí sos otra persona, no te conozco».

Esta situación permite un análisis desde diversas perspectivas. Por una parte, explicita algo circulante por el espacio institucional que tiene que ver con la falta de higiene, la suciedad y la podredumbre. Por otra, aparece un imaginario vinculado a las instituciones asilares, consideradas depósitos para «desechos humanos». Finalmente, podemos ver la dinamización transferencial de una representación de la Sra. H como «una bestia», «una loca desquiciada» y «que debe estar siempre en un manicomio».

Durante la supervisión exploramos, además, cómo el circulante motorizaba la contratransferencia del psicoterapeuta, quien se sentía «un monstruo», incapaz, incompetente, loco, «un kamikaze», por trabajar con la Sra. $\mathrm{H}$.

La articulación del imaginario social acerca de los centros psiquiátricos, del circulante institucional, de la historia de la paciente y de la movilización contratransferencial, nos permitió una mayor comprensión de lo acaecido para articular diferentes espacios y situaciones al servicio de una tarea compleja enmarcada en un dispositivo institucional. Sumar técnicas y dispositivos no es el caso. Articular es pararnos en un lugar con ciertas herramientas y, desde allí, ver qué está circulando y qué está transfiriéndose. 


\section{UN PSICOANÁLISIS ATENTO A LOS PROCESOS COLECTIVOS PARA SU ARTICULACIÓN}

Lo antedicho nos lleva a ser sensibles frente a diferentes contextos en los cuales las instituciones están inmersas y con los que se relacionan. En este marco amplio funciona el microuniverso institucional donde muchos trabajan. Otro elemento relevante refiere a la importancia de investigar detalladamente las circunstancias que rodearon la gestación de la institución: la historia de su creación y el desarrollo temporal con sus crisis. Esto aporta información para conocer sus fortalezas y fragilidades, sus equilibrios y desequilibrios, el vaivén entre los polos tanático y trófico que organizan el campo. Los contextos sociales, propios de cada época y lugar geográfico, van a dejar su huella en ocasión del surgimiento institucional.

En cuanto a los momentos de crisis y sus factores desencadenantes, estos nos proporcionan la pauta del grado de sinergia entre los polos trófico y tanático a la hora de ver lo ocurrido en un momento particular. Lo trófico es lo que sirve para organizar, producir sentido y construir e ir creando la tarea primaria de la institución. Lo tanático alude al ataque al pensamiento, lo destructivo y productor de caos y desorganización en el funcionamiento cotidiano del colectivo. En momentos saludables, ambos aspectos se reequilibran. Cuando se produce una crisis, hay un desbalance y el marco institucional deja de contener la faceta destructiva, que, liberada, entra a circular por los diferentes espacios institucionales, afectando a los sujetos más vulnerables y aptos para ser sus depositarios y chivos expiatorios. Los procesos colectivos son transindividuales. Su estudio, desde una óptica psicoanalítica, implica focalizarse en los puntos de entrecruzamiento institucionales. ${ }^{6}$

6 Freud (1988) hablaba del psicoanálisis como de una Spezialwissenschaft. Al decir de PaulLaurent Assoun (1982), esto refiere a que, para Freud, el saber está estructurado en compartimientos separados, cada uno de los cuales es una Spezialwissenchaft, que interacciona con otras en función de sus intereses recíprocos. 
Trabajar con esta perspectiva in mente nos coloca a la intemperie, ya que nuestro anonimato queda constantemente jaqueado por la propia situación. Las posibilidades de trabajar en la abstinencia y la frustración son muy escasas o nulas. Esto implica tener en cuenta lógicas muy diferentes a las usuales y hegemónicas. En tal sentido, debemos cuidarnos de no colonizar el psiquismo de los otros con nuestros propios puntos de vista sobre tópicos muy variados (religiosos, políticos, filosóficos, etc.), sino dejar que las decisiones corran siempre por cuenta de los involucrados.

La esperanza ante la posibilidad de producir cambios y transformaciones como consecuencia de nuestras intervenciones está siempre presente como un ideal a alcanzar, temperado por las inevitables desilusiones que habrá que procesar ante ciertos hechos de la realidad fáctica que nos muestran siempre tanto nuestros límites como nuestras limitaciones. ${ }^{7}$

\section{EL FOCO EN LA DIMENSIÓN INSTITUCIONAL}

La institución constituye el dispositivo macro que contiene espacios diversos, con diferentes encuadres, para llevar adelante un programa común, lo que debería estar adecuadamente articulado. Las instituciones configuran un dispositivo cuyo propósito es gestionar una tarea primaria, según la cual sirven de mediadoras con el conjunto de la sociedad en un cierto momento histórico (Bleger, 1985). Los seres humanos, por ser sociales, no podemos prescindir de ellas en nuestro relacionamiento con un mundo crecientemente complejo y globalizado en su funcionamiento.

Las instituciones no pueden desarrollar su tarea primaria sin contradicciones y conflictos. Su funcionamiento no es lineal ni carece de fisuras,

7 Freud (1991c) se ocupó de este tema al final de su vida en su texto Análisis terminable e interminable. La compilación de trabajos en torno a esta cuestión realizada por Sandler (1989) aporta una reflexión psicoanalítica actualizada. 
lo cual tiene efectos sobre los sujetos que interactúan en ellas. La mayoría de los psicoterapeutas ${ }^{8}$ hoy día trabajan tercerizados en el marco de las instituciones que componen el Sistema Nacional Integrado de Salud y de la ley 19.529 de Salud Mental, que ha sido parcialmente reglamentada. Aun cuando un psicoterapeuta atienda en su consultorio privado, siempre hay detrás una institución - por lo pronto, la institución de la psicoterapia-.${ }^{9}$ Esa institución virtual funciona como fondo de nuestra práctica psicoterapéutica y genera efectos sobre nosotros y nuestros pacientes. Hablamos de la presencia de las instituciones y de los propios psicoterapeutas en las redes sociales, con lo que allí dicen o dejan de decir. Lo mismo ocurre con el acontecer económico y cómo esto afecta nuestros aranceles.

Los potenciales pacientes, al consultar, ya llegan con información proveniente de diversas redes sociales y sitios de referencia en internet, lo que pone en jaque el proclamado anonimato y la reserva que supuestamente tiene el psicoterapeuta. Las redes sociales visibilizan temáticas que pueden llevar personas al consultorio de un psicoterapeuta determinado o bien generar que, estando en psicoterapia, las personas realicen planteos referidos a tópicos candentes (por ejemplo, abuso, violencia, diversidad sexual, etc.). Pensar todo esto desde nuestra disciplina configura un desafío porque nos obliga a ver dónde estamos parados y, así, a diferenciar el abordaje de estas cuestiones por disciplinas diferentes al psicoanálisis.

8 Desde la Federación Uruguaya de Psicoterapia se ha bregado por el reconocimiento de la profesión a la que se accede a través de una formación de posgrado. Al respecto, ver González Regadas (2008, 2010, 2015).

9 Nos referimos a la psicoterapia como una institución abstracta, que refiere a la psicoterapia y a los psicoterapeutas, pero también a las instituciones concretas que los nuclean y les sirven de marco. 
Si bien vamos a referirnos fundamentalmente a las instituciones vinculadas a algún tipo de cuidados, esto vale para examinar diferentes modelos, mirándolos psicoanalíticamente, para entender su funcionamiento y disfunciones. Hablar de lo circulante es aludir a un acontecer de la vida institucional que se da cotidianamente y que oscila entre cumplir con la tarea primaria o bloquearla por conflictos y contradicciones, lo que les provoca a sus integrantes un sufrimiento psíquico. Lo que designamos como enloquecedor o trasgresor es una experiencia o vivencia que se pone en marcha cuando se disparan ciertas vulnerabilidades institucionales ante una situación determinada y que están conectadas con algún conflicto o evento traumático acaecido en algún momento de su historia. En estos casos, exploramos el funcionamiento patológico y patogénico producido por la dimensión institucional, cuando se torna disfuncional y olvida la tarea primaria para la cual fue creada. Siempre ha sido un problema articular lo individual con lo grupal o institucional, ya que se los tiende a ver como contrapuestos o como si uno sustituyera al otro, cuando son, simplemente, diferentes.

En cualquier institución o grupo humano ocurre el fenómeno de la resonancia del circulante, ${ }^{10}$ que nos lleva a que dicha institución o grupo se configure efectivamente como algo que va a ponerse en movimiento y producir efectos en las personas involucradas. Podemos definir resonancia como aquello que un miembro privilegiado de un grupo promueve y tiene eco en los demás. Esto nos permite despersonalizar situaciones inherentes a la vida institucional y trabajarlas al servicio de la tarea primaria. Si

10 Didier Anzieu (1986) comenta que «En las situaciones de grupo, la vida psíquica intenta organizarse en torno a una fantasía individual, la de un miembro privilegiado o promotor, con relación al cual las fantasías de algunos miembros entran en resonancia» (pp. 142-143). Hay experiencias, vivencias, emociones y representaciones que tienen un alto poder evocativo para el resto de los integrantes del grupo y que son puestas en marcha por alguno de sus integrantes. 
bien el conflicto puede estar visiblemente localizado en una persona específica, se trata de algo en lo que todos participan de una forma u otra, lo que lo potencia. Por ejemplo, el temor individual a ser castigado por decir lo que se piensa y siente se puede mitigar dando certezas y seguridades acerca de los beneficios que tiene para todos la transparencia y la libertad de expresión puestas al servicio de la tarea en común.

Las instituciones padecen y hacen sufrir a sus integrantes aquellos problemas que aspiran a modificar. En función de esto, y en concordancia con Oury (1980), pensamos que la institución es funcional a la tarea primaria en la medida en que la propia institución sea analizada. Lamentablemente, esto no ocurre así y llega, incluso, a considerárselo como irrelevante. En nuestra experiencia, lo consideramos necesario para un funcionamiento eficaz. En tal sentido, sería bueno que las instituciones formadoras de psicoterapeutas implementaran espacios de análisis periódico de su funcionamiento para ver cómo impacta su acontecer durante el proceso de formación. ${ }^{11}$

\section{JOSSELYN: CIRCULANTES Y TRANSFERENCIAS INTERACTUANDO}

Un colega ${ }^{12}$ del interior del país recibe a una mujer de poco más de veinte años, derivada por orden judicial a causa de un proceso por acoso

11 Este era un tópico en el que insistía Harold Bridger. Al respecto, ver el capítulo xxI del libro Comunidad terapéutica y trastornos duales (González Regadas, 2001).

12 Agradecemos al colega que nos proporcionó este material, que ya tiene muchos años. Obviamente, está deformado para que no resulte posible reconocerlo. A propósito de este tópico, remitimos — porque nos parecen pertinentes - las Palabras preliminares de Freud (1978) al comienzo del caso de Dora, que dan cuenta de su proceder con respecto a este tópico (qué trasmitir y cómo hacerlo para que la clínica ilustre adecuadamente la teoría). 
y usurpación de identidad..$^{13}$ La paciente designada venía acompañada por una mujer mayor y, mientras se acercaban, una funcionaria le dice al colega: «iMirá quiénes te tocaron hoy!».

Después del encuentro con Josselyn, el psicoterapeuta señaló que la recepcionista le había hecho ese comentario porque «sabían todos» que la paciente tenía un proceso judicial en curso, información que, supuestamente, debía ser «absolutamente reservada». La madre de esta paciente era «una escandalosa» que tenía «podrido a todo el mundo»; iba con frecuencia a pedir «constancias y certificados para presentarle al juez». «Todos se la quieren sacar de encima, ella [la paciente] es una mentirosa y una ladrona», acotó la recepcionista.

Josselyn había sido vista por diferentes técnicos del equipo de salud mental. El psiquiatra que la derivaba le dijo al psicoterapeuta: «Hacé lo que puedas, pero no te preocupes porque es un trastornazo. ${ }^{14}$ Esto lo hacemos [la indicación de psicoterapia] para cumplir con el pedido del juez».

En síntesis, lo circulante por el centro de salud era que de Josselyn no podía esperarse mucho porque sus problemas eran de tal magnitud que era poco probable algún cambio en ella.

Durante las sesiones, al comienzo Josselyn se mostró muy reticente: apenas hablaba y miraba al terapeuta con mucho recelo, hasta que este aclaró de un modo explícito que no iba a informarle nada al juez, a menos que este lo solicitara, y que, en ese caso, previamente iba a compartir el eventual informe con ella. A partir de allí, la situación «aflojó un poco». Al cabo de un tiempo, el terapeuta «descubrió» que su paciente

13 Dicha identidad era electrónica, ya que el delito había sido usurpar la cuenta de una compañera de clase en una red social para, desde allí, enviarle mensajes acosadores a una docente de ambas. Esto salió a luz y la paciente debió afrontar un juicio y tratar su problemática psicológica por resolución del juez de la causa.

14 El psiquiatra estaba refiriéndose, «coloquialmente», al diagnóstico de trastorno fronterizo de la personalidad con un componente antisocial. 
tenía «escaso contacto con la realidad cotidiana», que distorsionaba groseramente «encarnando un personaje». Esto indujo al psicoterapeuta a indagar aspectos del personaje que actuaba y en qué momentos aparecía. Supo que había surgido en la adolescencia y que era una parasitación de la identidad de otra adolescente: «triunfadora, exitosa, querida por todos, agasajada». Esto configuraba la contracara de su realidad del momento: una joven esmirriada, fea, con un bajo rendimiento académico y rechazada por la mayoría de sus pares. La madre de crianza la había adoptado de un orfanato porque era hermana de otra niña a la que ella y su marido querían adoptar, «pero no podíamos separarlas». Esa otra hija (la hermana mayor de Josselyn) se convirtió, pasado el tiempo, en una profesional exitosa y con una familia propia.

Josselyn estudiaba para ser instrumentista quirúrgica. Una de sus docentes - a la que había acosado con mensajes y reclamos afectivos valiéndose de la identidad electrónica de una compañera de clase- coincidentemente trabajaba en el centro de salud donde Josselyn fue referida para asistirse por orden judicial. Esto no era una buena noticia para la paciente ya que, verosímilmente, podía «jugarle en contra».

A los seis meses de estar en psicoterapia, en una sala del centro de salud donde diversos estudiantes concurrían a hacer sus prácticas —entre ellos, Josselyn-, se constató la «desaparición de material de curaciones y medicamentos». Josselyn fue señalada como la responsable, «teniendo en cuenta sus antecedentes». La docente llevó «su caso» a las autoridades del centro de estudios. Como efectivamente tenía antecedentes, perdió su calidad de estudiante. Esto le produjo un tremendo impacto emocional y configuró una limitante en sus proyectos de autonomía personal y familiar, que fueron procesados en su psicoterapia en la institución. Al cabo de unos meses, luego de una laboriosa investigación administrativa realizada en el hospital, se identificó a la persona que había sustraído los materiales. En el centro de salud, en aquel entonces, había una «particular susceptibilidad» 
frente a cualquier tipo de robo, ya que había habido una seguidilla. En tal contexto y dados sus «antecedentes de mentirosa» — que previamente habían circulado por el centro asistencial—, Josselyn cargó con la sospecha de «ladrona» durante un período de seis meses. Al clarificarse la situación, el daño provocado por el rumor circulante ya estaba hecho. Su calidad de estudiante no le fue restituida a pesar de su reclamo.

Esta viñeta muestra cómo un circulante institucional (Josselyn «es una ladrona y mentirosa» y tiene una "madre escandalosa y pesada») coagula en ese momento en el sujeto más vulnerable, que oficia de chivo expiatorio para que la institución eluda las fallas notorias en sus mecanismos de control y uso de los materiales. La institución no era «vulnerable a los robos», sino que había «una ladrona». El peso recayó sobre Josselyn, quien, por su propia historia, fue la depositaria de lo trasgresor. Los chismeríos y rumores de pasillos la ubicaron en ese lugar. La vulnerabilidad institucional que dinamizó un circulante trasgresor encontró en Josselyn la excusa perfecta.

Durante las sesiones, la paciente transfirió su vulnerabilidad específica ante una situación de marginación, desvalorización y falta de reconocimiento, y mantuvo una postura reticente y suspicaz ante su psicoterapeuta. Esto la indujo a actuar, durante muchas sesiones, un personaje que era una impostura compensatoria para salir del lugar de mentirosa y ladrona en el que siempre quedaba ubicada. El personaje escogido fue el de una persona "exitosa, inteligente, que brillaba», como su hermana mayor. Trabajar durante un par de años esta problemática la llevó a una gradual desidentificación y abandono del personaje, lo que dio lugar a una identidad genuina sustentada en habilidades para el cuidado de niños. Esto la llevó a estudiar magisterio y a recibirse de maestra. Así, surgió una nueva opción laboral y dio un paso importante hacia una mayor autonomía personal. Su autoestima acrecentada fue sustituyendo una imagen devaluada de sí misma. 


\section{FUNCIONALIDAD Y DISFUNCIONES \\ EN LOS DISPOSITIVOS INSTITUCIONALES}

Cuando la institución funciona productivamente y el polo trófico contrabalancea al tanático, esta faceta trasgresora o enloquecedora es contenida en el marco instituido y no interfiere con la tarea primaria. El marco funciona como una madre lo suficientemente buena y con capacidad de ensoñación (Bion, 1966) para contener los temores que se le depositan, de forma tal que los individuos puedan asimilarlos provechosamente. Cuando predomina lo tanático, colapsa la función de contenedor del encuadre y lo allí depositado es devuelto en forma intacta (sin ser procesado psíquicamente) hacia los grupos más vulnerables y, de estos, hacia los individuos más proclives para recibirlos.

Los aspectos tróficos se vinculan, además, con las diferentes tareas que debemos afrontar durante el proceso de subjetivación o socialización. ${ }^{15}$ Cuando la institución recibe, incorpora, a una persona y le proporciona el sostén y la continencia necesarios, estamos en ese momento inaugural del proceso. De fracasar en esta etapa, se produce una falta básica (Balint, 1982). Luego, aparecen las instancias de reconocimiento de la singularidad y de las diferencias de quien fuera incorporado. El fracaso, por falta de empatía y frustraciones no adecuadas al momento, conlleva confusiones, simbiosis, amalgamas, seudoidentidades, etc. En momentos ulteriores, hay que encarar la instancia de redireccionar la propia existencia con base en lo aprendido, conformando metas, ideales, esperanzas e ilusiones para, finalmente, salir, egresar, de la institución (González Regadas, 2001).

15 Nos referimos al artículo «Bases para una psicopatología humanista», publicado en la Revista de psicoterapia psicoanalitica, de AUDEPP, y que actualizamos y ampliamos en forma electrónica (González Regadas, 2014). 


\section{REFERENCIAS BIBLIOGRÁFICAS}

AnZIEU, D. (1986). El grupo y el inconsciente. Lo imaginario grupal. Biblioteca Nueva.

Assoun, P. L. (1982). Introducción a la epistemología freudiana. Siglo XXI.

Balint, M. (1982). La falta básica. Aspectos terapéuticos de la regresión. Paidós.

Bion, W. R. (1966). Aprendiendo de la experiencia. Paidós.

Bleger, J. (1976). Psicohigiene y psicología institucional. Paidós.

BLEGER, J. (1985). El grupo como institución y el grupo en las instituciones.

Temas de psicología (Entrevista y grupos) (pp. 87-104). Nueva Visión.

Castoriadis, C. (1989). La institución imaginaria de la sociedad. Vol. 2: El imaginario social y la institución. Tusquets.

FREUd, S. (1978). Fragmento de análisis de un caso de histeria (1901[1905]).

En Obras completas (vol. vil, pp. 3-107). Amorrortu.

FREUD, S. (1979). Lo inconsciente (1915). En Obras completas (vol. XIV, pp. 153-213). Amorrortu.

FREUd, S. (1980). Esquema del psicoanálisis (1938[1940]). En Obras completas (vol. XXIII, pp. 133-209). Amorrortu.

FREUd, S. (1988). El interés por el psicoanálisis (1913). En Obras completas (vol. XIII, pp. 165-192). Amorrortu.

Freud, S. (1991a). Carta 52. En Obras completas (vol. I, pp. 274-279). Amorrortu.

FREUD, S. (1991b). Proyecto de psicología (1895). En Obras completas (vol. I, pp. 323-440). Amorrortu.

FreUd, S. (1991c). Análisis terminable e interminable (1937). En Obras completas (vol. xxiII, pp. 211-254). Amorrortu. 
GonzÁlez Regadas, E. M. (1992). La transferencia de lo psicótico en los grupos institucionales. Revista de psicoterapia psicoanalítica, III(4), 423-437.

GonzÁlez Regadas, E. M. (2001). Comunidad terapéutica y trastornos duales. Psicolibros.

GonzÁlez Regadas, E. M. (comp.) (2001). Drogas, ética y trasgresión. Psicolibros.

González Regadas, E. M. (2008). Psicoterapia, políticas públicas y sistemas nacionales de salud [ponencia]. VIII Congreso de la Federación Latinoamericana de Psicoterapia, San Pablo, Brasil.

GonzÁlez Regadas, E. M. (2009). Reflexiones desde el psicoanálisis de los procesos colectivos acerca de los pacientes con trastornos psicóticos y adicciones. Revista de psicoterapia psicoanalítica, VII(3), 41-55.

GonzÁlez RegadAs, E. M. (2009-2010). Integrando recursos técnicos en psicoterapia desde el psicoanálisis de los procesos colectivos [ponencia]. IX Congreso Latinoamericano de Psicoterapia, Lima, Perú. www.academia.edu/37072987/Integraci\%C3\%B3n_de_recursos _t \%C3\%A9cnicos_en_psicoterapia_psicoanal\%C3\%ADtica

GonzÁlez Regadas, E. M. (2010). Sin psicoterapia no hay psicoterapeuta. Sin psicoterapeuta no hay institución [presentación]. Primera actividad científica de la Federación Uruguaya de Psicoterapia, Montevideo: La psicoterapia del psicoterapeuta y su relación con los marcos institucionales. www.academia.edu/7646169/Sin_psicoterapia_no_hay_ psicoterapeuta_Sin_psicoterapeuta_no_hay_instituci\%C3\%B3n GonzÁlez RegadAs, E. M. (2014). Bases para una psicopatología humanista. Revista de psicoterapia psicoanalitica, VIII(3), 5-18. www.bvspsi.org. uy/local/TextosCompletos/audepp/025583272014080301.pdf GonzÁlez Regadas, E. M. (2015). Acreditación de la psicoterapia. Una salida para Uruguay [conferencia]. III Congreso Nacional de Federación Uruguaya de Psicoterapia y I Congreso Regional. 
GuatTaRI, F. (1976). Psicoanálisis y transversalidad. Siglo XXI.

OuRY, J. (1980). Algunos problemas teóricos de psicoterapia institucional. En M. Mannoni, Infancia alienada (pp. 119-132). Saltés.

SANDLER, J. (comp.) (1989). Estudio sobre el "Análisis terminable e interminable" de Sigmund Freud. Tecnipublicaciones. 